University of Texas at El Paso

ScholarWorks@UTEP

$1-2019$

\title{
Why Bilingualism Helps Autistic Children Function: A Symmetry- Based Explanation
}

Olga Kosheleva

The University of Texas at El Paso, olgak@utep.edu

Vladik Kreinovich

The University of Texas at El Paso, vladik@utep.edu

Follow this and additional works at: https://scholarworks.utep.edu/cs_techrep

Part of the Mathematics Commons

Comments:

Technical Report: UTEP-CS-19-01

Published in International Mathematical Forum, 2019, Vol. 14, No. 1, pp. 11-16.

\section{Recommended Citation}

Kosheleva, Olga and Kreinovich, Vladik, "Why Bilingualism Helps Autistic Children Function: A SymmetryBased Explanation" (2019). Departmental Technical Reports (CS). 1293.

https://scholarworks.utep.edu/cs_techrep/1293

This Article is brought to you for free and open access by the Computer Science at ScholarWorks@UTEP. It has been accepted for inclusion in Departmental Technical Reports (CS) by an authorized administrator of ScholarWorks@UTEP. For more information, please contact Iweber@utep.edu. 


\title{
Why Bilingualism Helps Autistic Children Function: A Symmetry-Based Explanation
}

\author{
Olga Kosheleva ${ }^{1}$ and Vladik Kreinovich ${ }^{2}$ \\ Department of ${ }^{1}$ Teacher Education and ${ }^{2}$ Computer Science \\ University of Texas at El Paso \\ 500 W. University \\ El Paso, TX 79968, USA \\ olgak@utep.edu,vladik@utep.edu
}

\begin{abstract}
One of the main problems of autistic children is that it is very difficult for them to switch to a different state, to a different activity - and such switches are often needed. Researchers have recently shown that bilingualism helps autistic children function: namely, it is somewhat easier for bilingual children to switch to a new activity. In this paper, we provide a possible explanation for this empirical phenomenon. Namely, we show that, in general terms, autism means difficulty with breaking symmetries of a state, and we describe how this general reformulation indeed explains the above recently discovered phenomenon.
\end{abstract}

\section{Formulation of the Problem}

What is autism: a brief reminder. Autism is defined as a "developmental disorder characterized by difficulties with social interaction and communication, and by restricted and repetitive behavior"; see, e.g., $[1,7]$.

Researchers are trying to help. At present, there is no way to completely cure this disorder. However, there are many techniques that help some patients function better - i.e., make it easier for them to switch to a new activity and/or easier to disrupt the repetitive behavior.

Can bilingualism help? There is plenty of evidence that, in general, bilingualism - i.e., knowing two languages - helps in problem solving; see, e.g., $[2,3]$. Because of this general fact, researchers studying autism decided to check whether a similar improvement can be observed in autistic children.

It helps but why? The results of this research [5] show that bilingualism indeed statistically significantly helps bilingual children switch to a new activity: on average, bilingual autistic children performed better on such switching tasks than monolingual ones. These results confirmed anecdotal evidence listed in [6]. 
Since knowing two languages helps autistic children function, it seems desirable to recommend raising such children in a two-language environment. Of course, biligualism does not make these children fully overcome their limitations, it is not a miracle cure, but still, such an improvement is important in situations when any improvement is welcome.

What is not very clear is why such an improvement occurs. In this paper, we provide a possible general explanation.

\section{How Can We Describe Autism in Precise Terms?}

What kind of an explanation do we want. With respect to behavioral phenomena, the word "explanation" may mean different things. What we are interested in is an explanation which is as general as possible. Let us explain on a simple example what we have in mind.

As an example, let us take a known phenomenon: that in the extreme-stress situations, e.g., on the battlefield, people sometimes involuntarily urinate or defecate (and animals, by the way, exhibit the same behavior). From the physiological viewpoint, a possible explanation would mean explaining how signals travel via nerves, what chemicals are released, and how the resulting chain of events leads to defecation. This physiological picture is important to know, it provides a clear explanation of how exactly this phenomenon occurs, but it does not explain why nature adopted this phenomenon in the first place. In other words, such a specific (non-general) explanation would enable us to predict that creatures with a similar physiological system would probably exhibit the same behavior, but it will not enable to say anything about possible behavior of creatures whose physiology is different.

A general explanation is that in extreme stress situations, the body mobilizes all its resources, all its energy, all its ability to deal with a potentially harmful stressor. From this viewpoint, spending energy of digesting food does not help: for example, if we need to run away from a lethal predator, the faster we run, the better our chances to survive. Getting rid of all the food prevents us from wasting resources on digesting this food, and enables us to concentrate on what is really important in this situation: one's survival.

Based on this general explanation, we can easily predict that whatever the physiology, all living species should exhibit a similar behavior.

To come up with a general explanation, let us reformulate the problem in general terms. In order to come up with an explanation for the above bilingual-autism phenomenon which is as general as possible, we need to describe our problem in terms which are as general as possible.

Our problem involves two phenomena: autism and bilingualism. Let us therefore try to reformulate these two phenomena in terms which are as general as possible. 
Let us describe autism in most general terms. As we have mentioned earlier, autism means, in effect, two phenomena:

- When an autistic person is in a certain state, a state appropriate for a certain situation, it is much more difficult for this person - in comparison with non-autistic ones - to switch to a different state. For example, when such a child is at home, it is difficult to convince him or her to go outside. If an autistic child came heavily dressed from the outside, it is difficult to convince him/her to change to lighter home clothes, etc.

- Also, autistic children tends to get involved in repetitive behavior. For them, it is not easy to stop such a behavior and switch to a different activity.

How can we describe these two phenomena in general terms?

The first phenomenon start with a person in the same state - an autistic person would prefer to stay in this state. What does it mean "staying in the same state"? It means that the state does not change with time, i.e., if we go from the original moment $t$ to a new moment $t+t_{0}$, the state does not change. In other words, it means that the state does not change ( $=$ is invariant) under any time shift: $t \rightarrow t+t_{0}$.

Such an invariance with respect to a transformation is also known as symmetry (see, e.g., $[4,8]$ ), because what we call symmetries in real life is, actually, invariance with respect to some transformation. For example, spherical symmetry means that no matter how we rotate a spherical object, i.e., whatever rotation transformation we apply, the object remains the same. Similarly, invariance relative to rotations around a fixed axis means that rotations around this axis do not change the object. In these terms, being in the same state means that the state is symmetric with respect to time shifts.

From this viewpoint, changing the state means breaking this symmetry. In these terms, the first phenomenon means that for autistic children, it is more difficult to break this time-shifting symmetry than for non-autistic children.

Similarly, repetitive behavior means that the child's actions are repeated again and again. If we denote the duration of one cycle of such actions by $t_{0}$, this means that the child's state repeats itself after the time interval $t_{0}$. In other words, for every moment $t$, the state at this moment $t$ and the state at the moment $t+t_{0}$ - corresponding to the similar stage in the next cycle - are practically the same.

Thus, here we also have a symmetry with respect to a time shift $t \rightarrow t+t_{0}$. The only difference from the first phenomenon is that:

- in the first phenomenon, we had symmetry with respect to all possible time shifts, with all possible values $t_{0}>0$, while

- in the second phenomenon, we only have symmetry for one specific time shift $t_{0}$ (and, of course, as a consequence, for time shifts $2 t_{0}, 3 t_{0}$, etc.

Here, autistic behavior means that it is difficult for a child to stop this repeated activity - and thus, to break the original symmetry. 
Summarizing, we can say that autistic activity means difficulty with breaking symmetry, i.e., difficulty of switching from a symmetric state or behavior to a state or behavior that does not have this symmetry.

\section{This Reformulation Explains Why Autistic Children Get Involved in Repetitive Activi- ties in the First Place}

Let us show that the above general reformulation of the autism phenomenon explains the important part of the second phenomenon of autism - the part that we previously took for granted - that autistic children get involved in repetitive activities in the first place.

Indeed, in the first phenomenon, difficulty to break symmetry means that it is difficult to immediately switch from the original state, in which we had invariance with respect all possible shifts, to a state with a change in time, in which we have no symmetries at all.

From this viewpoint, it may be easier to change to a state in which some symmetries remain - i.e., to a new state which may not be invariant with respect to all possible shifts, but which is invariant with respect to some shifts $t_{0}$. And such new states are exactly states with repetitive behavior!

This is similar to physics, where, according to statistical physics, it is not very probable to go from a highly symmetric state immediately to a state with no symmetries at all: usually, first, some symmetries are broken but some other symmetries remain.

For example, as we heat a solid body, we usually do not immediately go from a highly symmetric crystal structure of a solid body to a completely nonsymmetric state of a gas: first, we usually go through the phase of a liquid, where some invariance remains - e.g., the density remains invariant with respect to all the shifts; see, e.g., $[4,8]$.

\section{What Does Bilingualism Means in These Terms and How This Explains Why It Is Eas- ier for Bilingual Autistic Children to Switch to a Different Activity}

What does bilingualism means in practice. Active bilingualism means that a person constantly switches from one language to another - depending on who he talks to. For example, in a bilingual family, a child may prefer to use one language with the mother and another with the father - or, as typical in immigrant families in the US, one language (English) with assimilated parents and another language (language of the old country) with the grandparents. 
What does bilingualism mean in symmetry terms. The constant switching means that even the seemingly stable state is not as symmetric as with monolingual kids: other parameters describing this state may remain the same, but the language of use when talking and/or listening changes rather unpredictably all the time - and talking and listening are definitely important parts of human activity.

This explains why bilingualism helps autistic children. Indeed, in our terms, the main problem of autistic children is that for them, it is much more difficult (than for other children) to switch from a symmetric state to a state with fewer symmetries - and the more symmetries need to be broken, the more difficult the transition.

As we have mentioned, even in seemingly the same state, the actual state of a monolingual child is more symmetric than the state of a bilingual one. Thus, for a monolingual child, transition to a new state means breaking more symmetries than for a bilingual one. For autistic children, the more symmetry violations, the more difficult the transition. So, this explains why a transition to a new state is somewhat more difficult for monolingual autistic children than for bilingual ones.

\section{Acknowledgments}

This work was partially supported by the US National Science Foundation via grant HRD-1242122 (Cyber-ShARE Center of Excellence).

\section{References}

[1] "Autism Spectrum Disorder", 299.00 (F84.0). In: American Psychiatric Association. Diagnostic and Statistical Manual of Mental Disorders, Fifth Edition, American Psychiatric Publishing, 2013.

[2] E. Byalystok, F. Craik, and G. Luk, "Cognitive control and lexical access in younger and older bilinguala", Journal of Experimental Psychology: Learning, Memory, and Cognition, 2008, Vol. 34, Publ. 859.

[3] E. Byalystok and M. M. Martin, "Attention and inhibition in bilingual children: evidence from the dimensional change card sort task", Developmental Science, 2004, Vol. 7, pp. 325-339.

[4] R. Feynman, R. Leighton, and M. Sands, The Feynman Lectures on Physics, Addison Wesley, Boston, Massachusetts, 2005.

[5] A. M. Gonzalez-Barrero and A. S. Nadig, "Can bilingualism mitigate setshifting difficulties in children with Autistic Spectrim Disorders", Child Development, 2017. 
[6] E. Kay-Raining Bird, F. Genesee, and L. Verhoeven, "Bilingualism with children with developmental disorders: a narrative review", Journal of Communication Disorders, 2016, Vol. 63, pp. 1-14.

[7] National Institute of Mental Heath (NIMH), Autism Spectrum Disorder, https://www.nimh.nih.gov/health/topics/autism-spectrum-disordersasd/index.shtml, retrieved in January 2018.

[8] K. S. Thorne and R. D. Blandford, Modern Classical Physics: Optics, Fluids, Plasmas, Elasticity, Relativity, and Statistical Physics, Princeton University Press, Princeton, New Jersey, 2017. 